$15^{\text {th }}$ International Conference on

AEROSPACE SCIENCES \& AVIATION TECHNOLOGY,

$\boldsymbol{A S A T}$ - 15 - May 28 - 30, 2013, Email: asat@mtc.edu.eg, Military Technical College, Kobry Elkobbah, Cairo, Egypt, Tel: +(202) 24025292 -24036138, Fax: +(202) 22621908

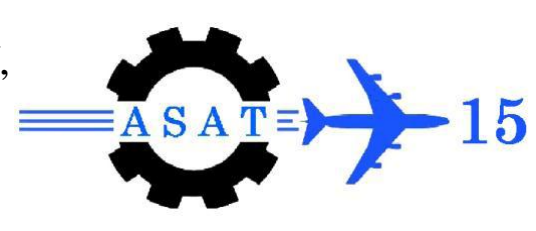

\title{
Antenna Beamforming using New Blind Least Mean Square for Wideband Interference Cancelation
}

\author{
\{M. S. Alsholle*, M. A. Abdalla, H. A. Elregily, and A. A. Mitkees $\}^{\dagger}$
}

\begin{abstract}
This paper presents the application of an antenna beamforimging using a modified and new blind adaptive algorithm for nulling of interference wideband signals. An adaptive linear array antenna is employed. The theoretical design principle of the proposed algorithm is presented. The results confirm that the proposed algorithm achieves better antenna array beamformation nulling compared to conventional algorithms. The proposed array has $6 \mathrm{~dB}$ interference signals nulling better compared to conventional least mean square algorithm and does not need a reference signal.
\end{abstract}

Keywords: Beamforming, interference cancelation, CMA, LMS , AWGN

\section{Introduction}

The continued demand for wireless communication services is spearheading research in new techniques for enhancing spectral utilization. One such technique is the use of adaptive or smart antennas to produce a movable beam pattern that can be directed to the desired coverage areas. This characteristic minimizes the impact of unwanted noise and interference, thereby improving the quality of the desired signal.

An adaptive antenna consists of an array of antenna elements. The signals picked up by these individual elements are combined through the use of a signal processing unit to form a beam pattern that can be steered toward the desired coverage direction [1]. This can be done by using an adaptive control processor to change the weights of the array antenna elements. Adaptive array is an antenna system that can modify its beampattern or other parameters, by means of internal feedback control while the antenna system is operating. Most adaptive algorithms are derived by first creating a performance criterion, and then generating a set of iterative equations to adjust the weights such that the performance criterion is met. For this purpose, an adaptive antenna array [2] may change the patterns automatically in response to the signal environment. Adaptive arrays are also known as adaptive beamformers, or adaptive antennas.

The Least Mean Square (LMS) and Constant Modulus Algorithm (CMA) are two commonly used algorithms for adaptive beam forming. The former has good tracking performance with low computational complexity, and is robust against coherent signals. On the other hand, the LMS algorithm can achieve a faster convergence and more rebuts but it needs reference signal. This reference signal is not available in some compunctions system [1].

\footnotetext{
* engineer_alshole @yahoo.com

$\dagger$ Egyptian Armed Forces, Egypt.
} 
In this paper, we introduce an interference cancelation for an adaptive array antenna using a new adaptive algorithm. The new algorithm was designed by modifying the conventional LMS through the CMA algorithm. The new algorithm is called LMS-CMA and it combines the advantages of the LMS and CMA algorithms.

\section{Theory}

Assume the adaptive $\mathrm{M}$ element linear array antenna with constant spacing between the elements (d). Let $\phi$ be the direction of arrival of the incident planer wave front as shown in Fig. (1). The incident signal is a wideband signal $S(t)$, the additive noise is $N(t)$, and the steering matrix of the array antenna is $\mathrm{A}(\phi)$. Thus, the received signal matrix $\mathrm{X}$ is [3].

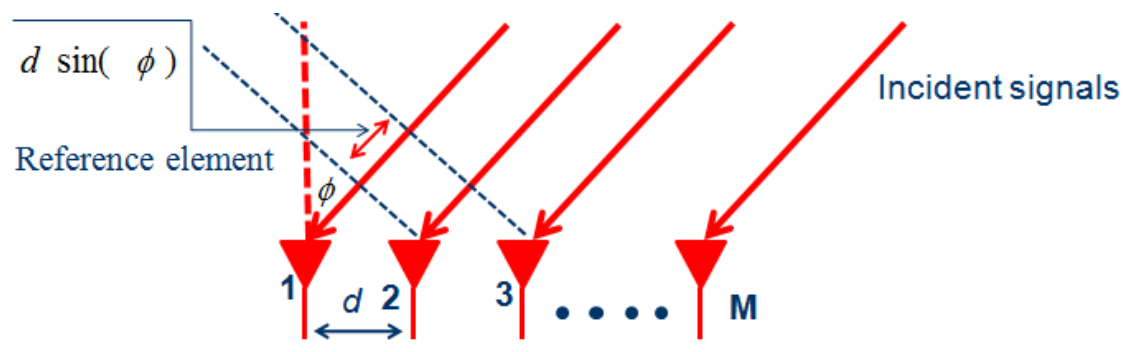

Fig. 1 An M element Linear Uniform Array Antenna

If the first element is taken as a reference point, thus, the phase difference between the $\mathrm{m}^{\text {th }}$ element output and the first one is given by

$$
\varphi_{m}=\frac{2 \pi}{\lambda_{c}}(M-1) d \sin (\theta)
$$

Where $\lambda c$ is the operating wavelength, $d$ is the distance between array elements. So, for a single incident wavefield in the noise free case, the signal received at the $\mathrm{mth}$ sensor is

$$
\mathbf{x}_{l}(f, k)=s\left(t, \tau_{l}\left(\theta_{1}\right)\right) \cos \left(\omega_{c}\left(t-\tau_{l}\left(\theta_{1}\right)\right)+\varphi\right) \quad \text { for } \quad 1 \leq l \leq m
$$

where $s(t)$ is the baseband source signal, $\omega c$ is the center frequency, $\theta 1$ is the azimuth angle $\tau l$ is the time delay associated with the 1 sensor signal and $\phi$ is the phase angle.

This signal can be expressed in the frequency domain as

$$
X_{l}(\omega)=S(\omega) e^{-j \phi} e^{-j\left(\omega-\omega_{c}\right) \tau_{l}\left(\theta_{1}\right)} \quad \text { for } \quad 1 \leq l \leq m
$$

where $S(\omega)$ denotes the Fourier transform of the source signal $s(t)$.

In the case of multiple incident wavefields, for example there are $\mathrm{n}$ wavefields incident on the array, the superposition principle indicates that the mth array sensor signal is defined by the linear combination of the separate effects of the individual wavefields. Therefore the data model in the presence of environmental noise takes the form

$$
X_{l}(\omega)=\sum_{k=1}^{n} S_{k}(\omega) e^{-j \phi_{k}} e^{-j\left(\omega-\omega_{c}\right) \tau_{l}\left(\theta_{k}\right)}+N_{l}(\omega) \quad \text { for } \quad 1 \leq l \leq m
$$

where $\operatorname{Sk}(\omega)$ denotes the Fourier transform of the kth source signal $\operatorname{Sk}(\omega)$. and $\mathrm{Nk}(\omega)$ denotes the noise of the mth sensor. It is assumed that the noise samples are uncorrelated with the signals in the matrix form, the model is defined by 


$$
x(\omega)=A(\omega, \theta) s(\omega)+n(\omega)
$$

where $\mathrm{x}(\omega)$ is the $\mathrm{m} \times 1$ spectral array output vector, $\mathrm{A}(\omega, \theta)$ denotes the $\mathrm{M} \times \mathrm{d}$ steering matrix, $s(\omega)$ is the $\mathrm{d} \times 1$ spectral source vector and $\mathrm{n}(\omega)$ denote the $\mathrm{m} \times 1$ noise vector so that

$$
\begin{gathered}
x(\omega)=\left[\begin{array}{llll}
X_{1}(\omega) & X_{2}(\omega) & \ldots \ldots \ldots & X_{m}(\omega)
\end{array}\right]^{T} \\
s(\omega)=\left[\begin{array}{llll}
S_{1}(\omega) & S_{2}(\omega) & \ldots \ldots \ldots & S_{d}(\omega)
\end{array}\right]^{T} \\
n(\omega)=\left[\begin{array}{llll}
N_{1}(\omega) & N_{2}(\omega) & \ldots \ldots \ldots & N_{m}(\omega)
\end{array}\right]^{T} \\
A(\omega, \theta)=\left[\begin{array}{llll}
a_{1}\left(\omega, \theta_{1}\right) & a_{2}\left(\omega, \theta_{1}\right) & \ldots \ldots \ldots . . & a_{d}\left(\omega, \theta_{1}\right)
\end{array}\right]^{T}
\end{gathered}
$$

where

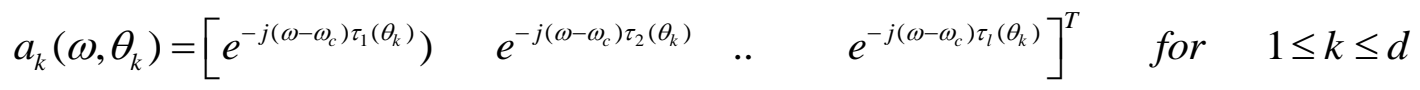

\section{Adaptive Beamformer for Wideband Signals}

Most of adaptive beamforming algorithms may be categorized into two classes according to whether a training signal is used or not. One class of these algorithms is the non-blind adaptive algorithm in which a training signal is used to adjust the array weight vector. Another technique is to use a blind adaptive algorithm which does not require a training signal. Since the non-blind algorithms use a training signal, during the training period, data cannot be sent over the radio channel. This reduces the spectral efficiency of the system [4].

In the following subsections, we introduce the basic principles of the LMS and CMA algorithms and our new modified LMS-CMA one. Also, we will assume that there are M isotropic element adaptive linear array, which employs LMS-CMA as its beam forming algorithm. Let the desired signal $\mathrm{d}(\mathrm{t})$ and a interference $\mathrm{s}(\mathrm{t})$, both originated from a distance, are impinging on the array at an angle $\theta \mathrm{d}$ and $\theta \mathrm{i}$, respectively, as shown in Fig. 1. For the following described adaptive beamformer, the output y (k), and weighted functions w (k) used in the LMS algorithm by the following three equations [5].

$$
\begin{gathered}
y(k)=\mathbf{w}^{H} \mathbf{x}(k) \\
\mathbf{w}(k+1)=\mathbf{w}(k)+\mu \mathbf{x}(k) \varepsilon^{*}(k)
\end{gathered}
$$

where $\varepsilon(\mathrm{k})$ is the error that can be derived differently in each algorithm.

\subsection{Non-blind Adaptive LMS Beamforming Algorithm}

In a non-blind adaptive algorithm, a training signal, $d(t)$, which is known to both the transmitter and receiver, is sent from the transmitter to the receiver during the training period. The beamformer in the receiver uses the information of the training signal to compute the optimal weight vector, wopt. After the training period, data is sent and the beamformer uses the weight vector computed previously to process the received signal. If the radio channel and the interference characteristics remain constant from one training period until the next, the weight vector wopt will contain the information of the channel and the interference, and their effect on the received signal will be compensated in the output of the array. A number of weight adjustment procedures or algorithms exist which minimize the mean square error. Minimization is usually accomplished by gradient search techniques. Gradient algorithms 
assume an established quadratic performance surface which is a function of the array weights, the performance surface $\mathrm{J}\left(\mathrm{W}^{\prime}\right)$ is in the shape of an elliptic parabola having one minimum.

One method that has been proven to be very useful is the LMS algorithm. LMS algorithm is a gradient based quadratic approach. The LMS algorithm for beamforming depends on a storing reference signal at the receiver. This signal is predefined before the transmission. The task of the algorithm is to minimize the error between the received signal and the reference signal. The non-iterative algorithms use a training signal, during the training period, data cannot be sent over the radio channel. The algorithm of the LMS algorithm for beamforming the array pattern is shown in Fig. 2.

By minimizing error between the reference signal $d(t)$ (which closely matches or has some extent of correlation with the desired signal estimate) and the beam former output $y(t)$ equal to $\mathrm{W}(\mathrm{t})$. This is a classical wiener filtering problem for which solution can be iteratively found using the LMS algorithm. The error $\varepsilon(\mathrm{k})$ in the LMS algorithm is [5].

$$
\varepsilon(k)=d(k)-y(k)
$$

where $\mathrm{d}(\mathrm{k})$ is reference signal.

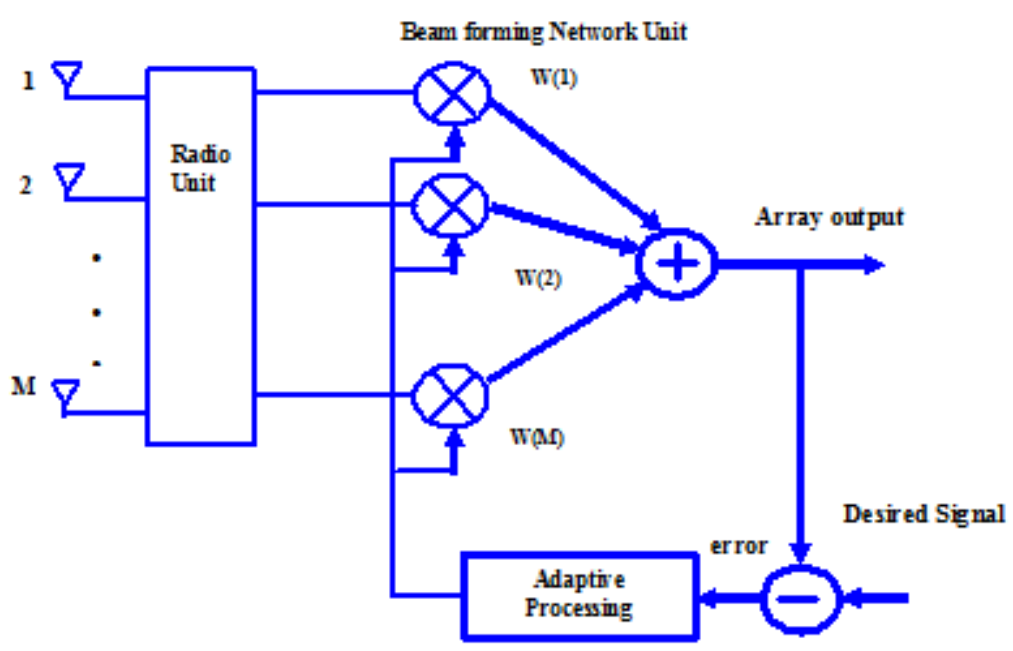

Fig. 2 A LMS algorithm

\subsection{Blind Adaptive CMA Beamforming Algorithm}

Blind adaptive algorithms do not require a training sequence. Instead, they exploit some known properties of the desired received signal. The most popular blind algorithms is basically based on estimation the direction of arrival of the received signals. One of the most known blind algorithms is the constant modulus algorithm which can recover one signal from multiple incident signals without using reference signal. [6] The CMA adjusts the weight vector of the adaptive array to minimize the variation of the envelope at the output of the array. After the algorithm converges, the array can steer a beam in the direction of the signal of interest (SOI), and nulls in the directions of the interference. The block diagram of CMA algorithm is shown in Fig. 3. 


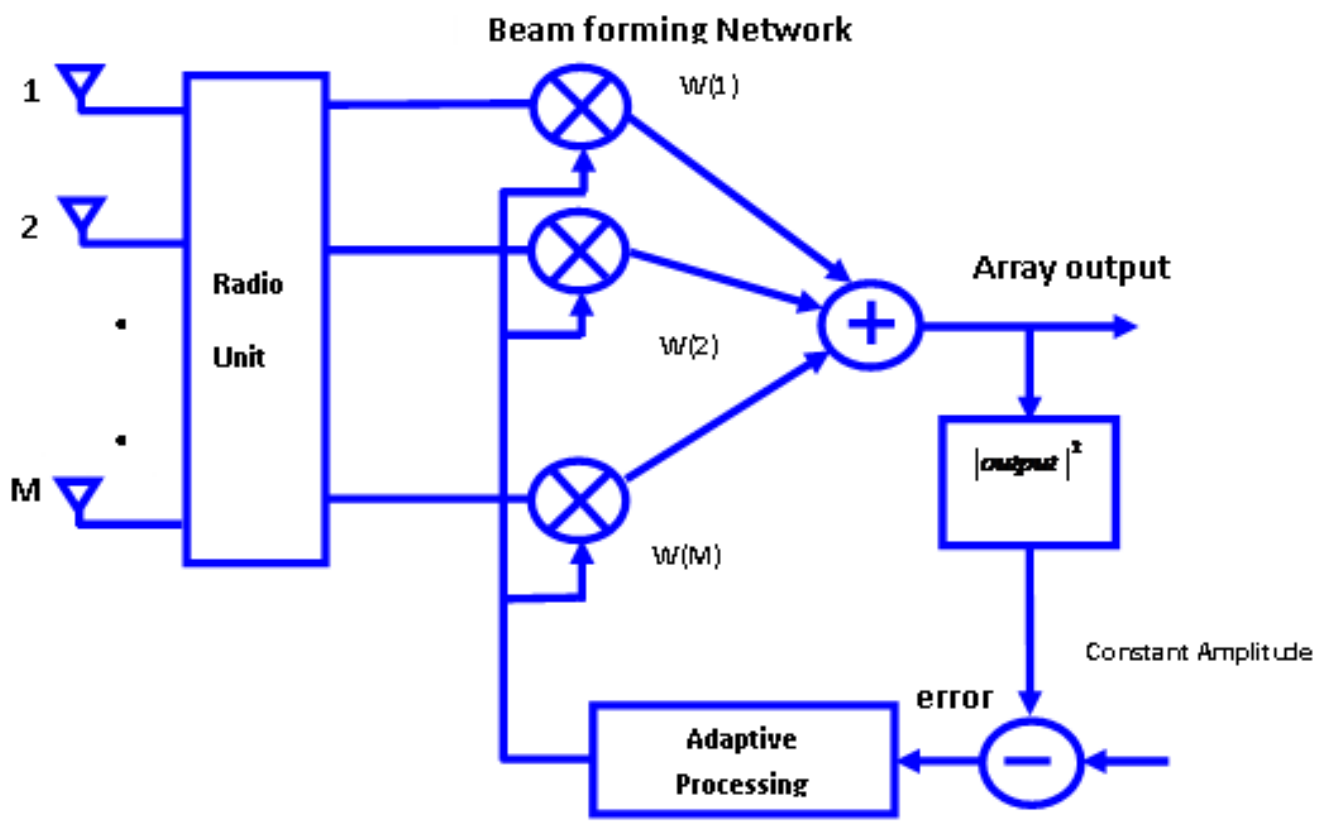

Fig. 3 CMA adaptive beamforming

The error signal in the CMA algorithm is [6].

$$
\varepsilon(k)=|y(k)|^{2}-1
$$

Similar to LMS, but with update error $\left(|y(k)|^{2}-1\right)$, and the desired signal is estimated by $s(k)=1$. The greatest drawback of the CMA is its relatively slow convergence which becomes ever more significant as wireless applications involving rapid changes in the channel characteristic become more prominent. Various enhancements of the CMA offering improved convergence have been investigated, but most involve significant increases in complexity or computational cost.

\subsection{Modified LMS-CMA Algorithm for Wideband Interference Cancellation}

In this subsection, the proposed version of LMS-CMA as the solution of the convergence problem is described. The modified LMS-CMA is based simply on output of CMA as reference signal for LMS. The modified block diagram is shown in Fig. 4. According to Fig. 4, the refernce signal stage of the LMS-CMA scheme is based on the CMA algorithm with its weight vector at the $(j+1)$ th iteration updated according to [4]. The error signal has been then obtained on this new modification as given in (13).

$$
\varepsilon(k)=d_{C M A}(k)-y(k)
$$

where $d_{C M A}(k)$ is output of CMA beamformer as the reference signal for LMS beamformer 


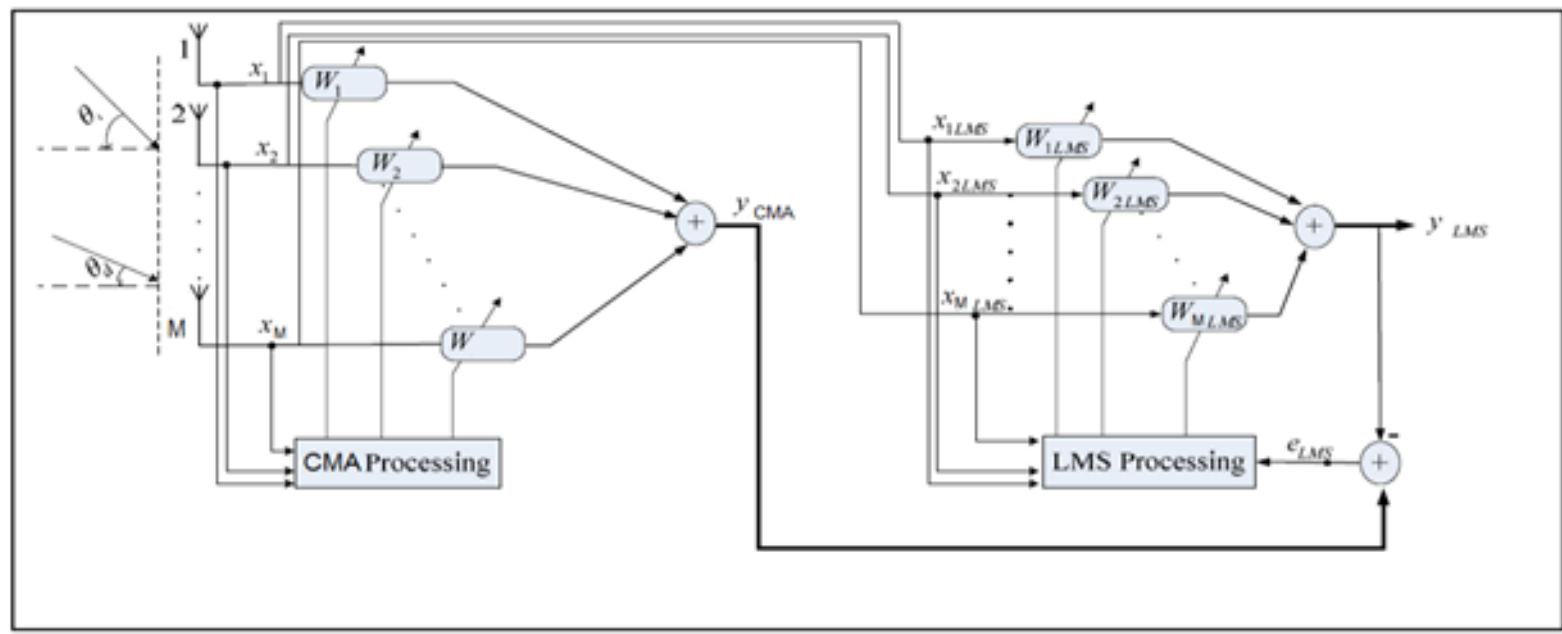

Fig. 4 The block diagram of an adaptive array system employing the LMS-CMA algorithm

\section{4- Results and Discussions}

In this section we introduce the performance of the modified LSM-CMA algotithim for interference minimization for a wide band signal. In the studied case, two interference signals are located at $-20,20$ degree from the array bore sight and one desired signal is located at $0 \mathrm{o}$ from the array bore sight present in the instantaneous view of the uniform linear antenna array. The antenna array consists of 11 elements and the spacing between them is considered to be half of the wave length corresponds to the used carrier frequency. Also, it was assumed the presence of AWGN of zero mean and variance $\sigma 2$. All the tap weights are initially set to zero. The interference cancelation has been done using the non blind LMS algorithm, the blind CMA algorithm and our modified blind LMS-CMA algorithm. The simulated results for the interference cancelation using blind LMS is shown in Fig. 5. From Fig 5, it is clear that the LMS beamformer has successfully nullified the unwanted incident signals at directions 20, 20 degree. We conclude that the LMS beamformer fails nullify the unwanted signals if require reference signal which may not be available in some applications.

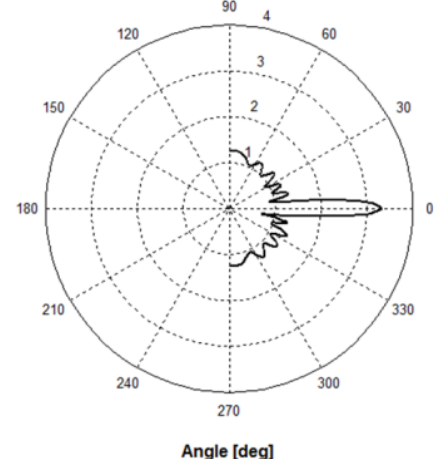

(a)

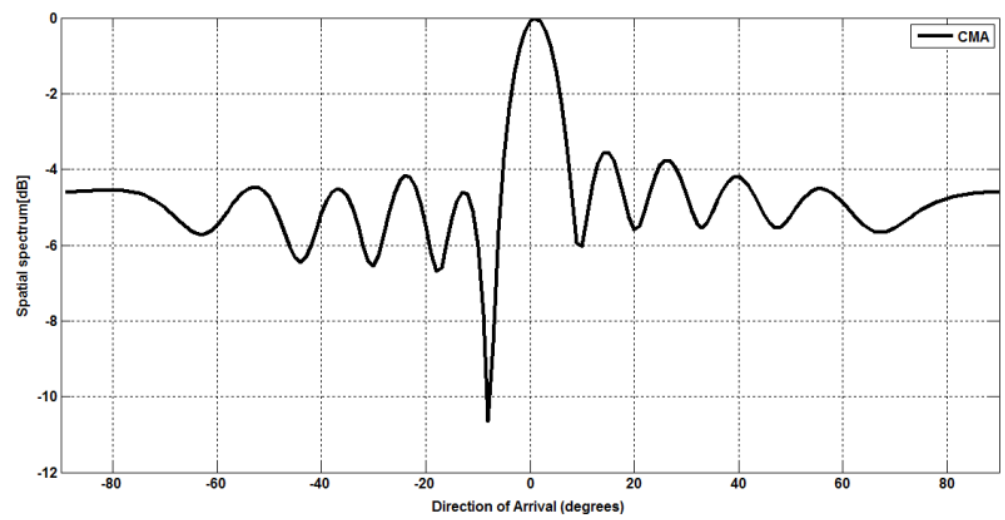

(b)

Fig. 5 The LMS beamformer (a) The adaptive antenna array pattern (b) The spatial spectrum. 
To overcome this problem, Modules Algorithm (CMA) is applied. The performance evaluation of the Modules Algorithm (CMA) as Blind Algorithms is presented in next subsection. The previous problem was investigated using the CMA algorithm and the spatial spectrum In the simulation we considered the same assumptions that we have taken before in the simulation of the LMS beamformer. The results are shown in Fig. 6 from which we can conclude that the CMA has successfully nullified the unwanted incident signals at directions -20 o, 20o. However, comparing these results to the LMS results shown in Fig. 6, it is clear that, the LMS achieves better performance than CMA. However, it is worth to mention that the CMA is non blind algorithm.

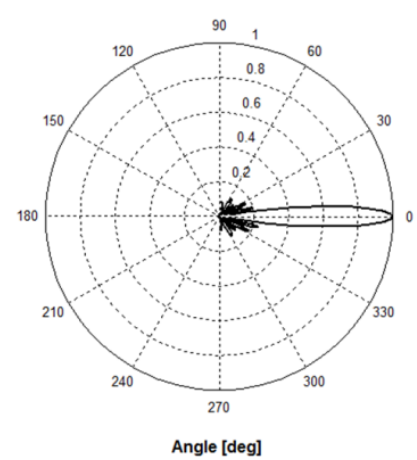

(a)

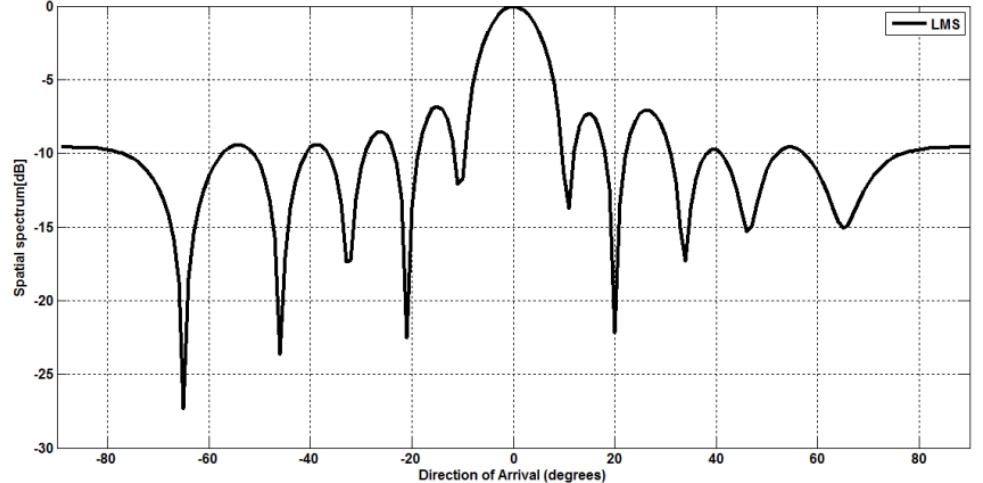

(b)

Fig. 6 The CMA beamformer (a) The adaptive antenna array pattern (b) The spatial spectrum.

To illustrate the capability of the proposed LMS- CMA algorithm, the previous interference problem was re investigated using our modified algorithm. A comparison between the spatial spectrum of received signal of the adaptive beamformer using LMS, CMA, and modified LMS-CMA algorithms is shown in Fig. 8 and Fig 9 for two different random interference signals. From the figures, we can claim that the modified blind LMS-CMA achieves superior performance than LMS and CMA algorithms, respectively.

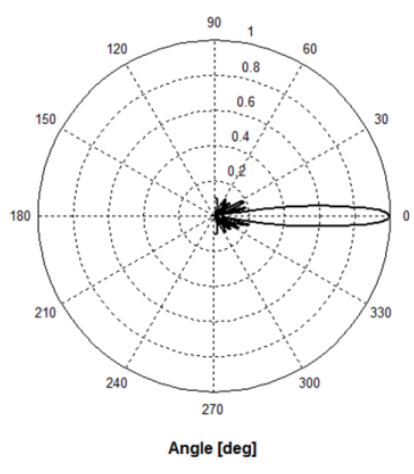

(a)

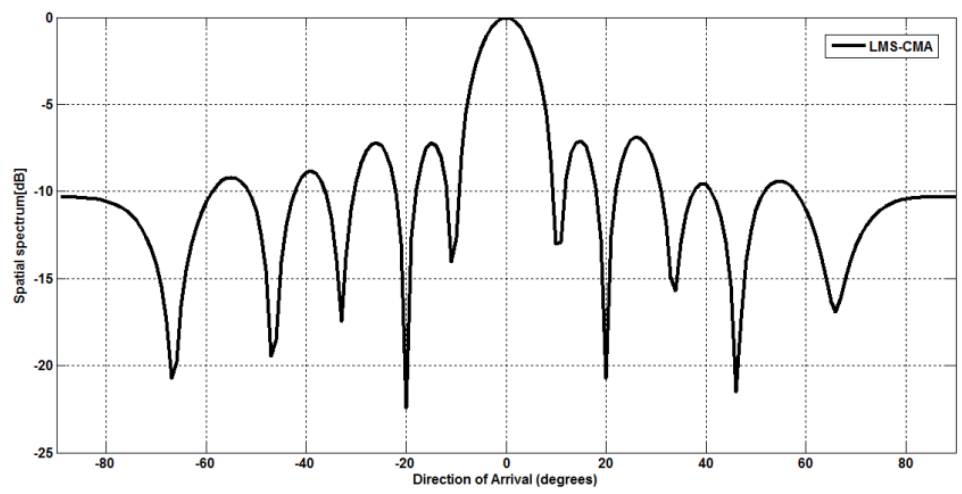

(b)

Fig. 7 The LMS-CMA beamformer (a) The adaptive antenna array pattern (b) The spatial spectrum. 


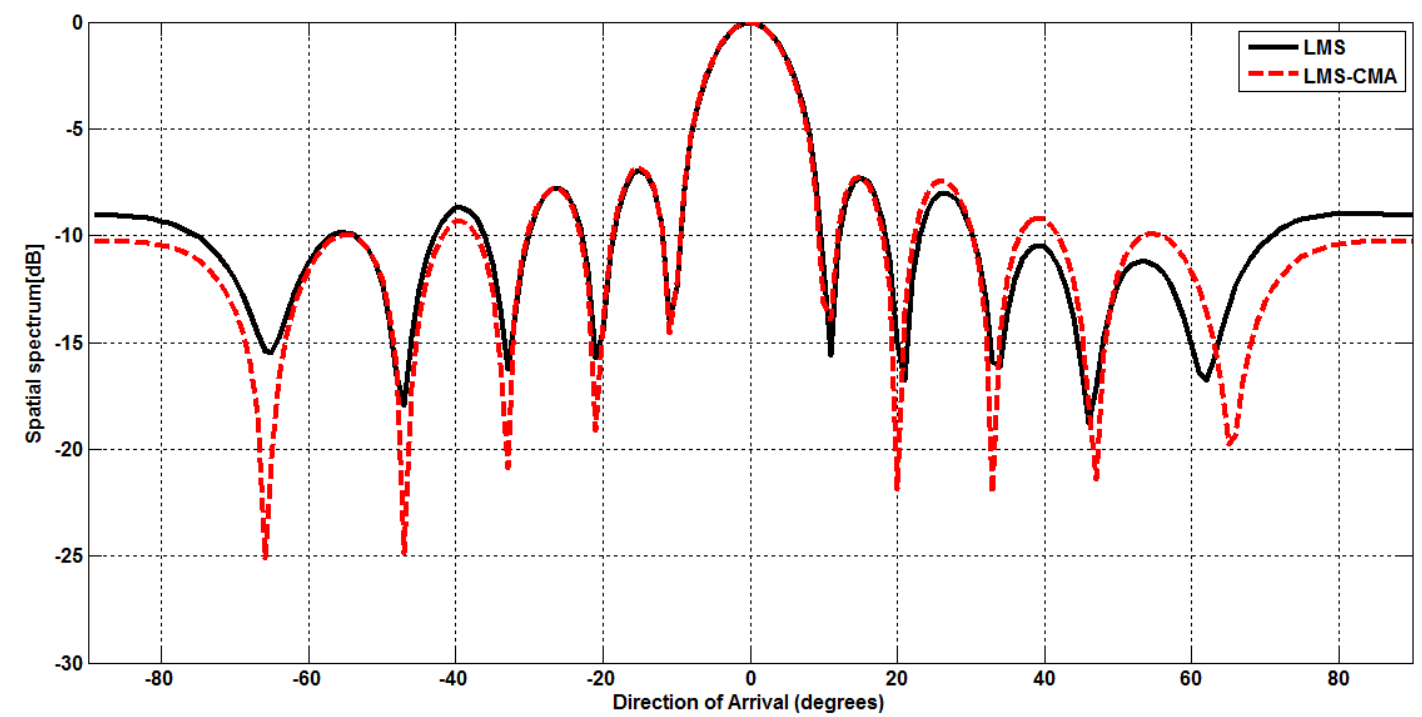

Fig. 8 A comparison between the spatial spectrum of received signal of the adaptive beamformer using LMS and modified LMS-CMA algorithms

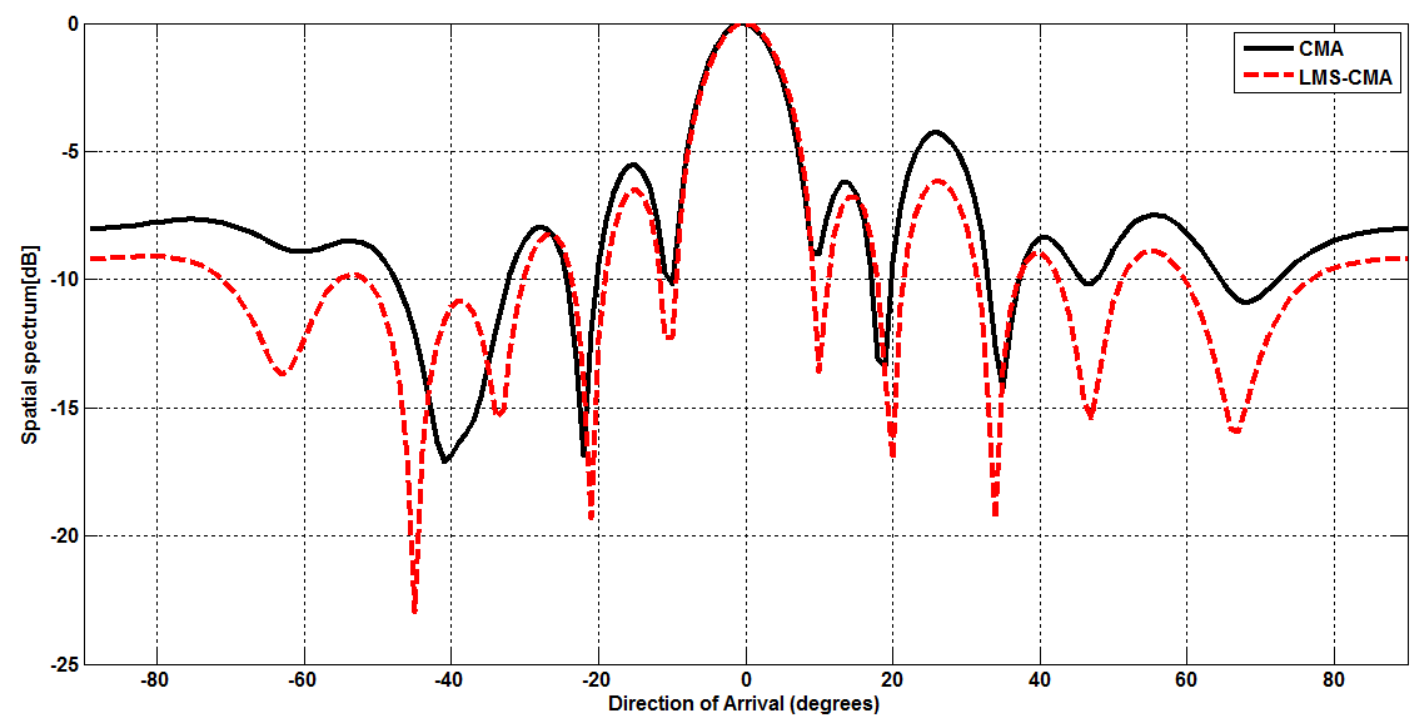

Fig. 9 A comparison between the spatial spectrum of received signal of the adaptive beamformer using CMA and modified LMS-CMA algorithms

\section{Conclusions}

An antenna beamforimging using a new LMS-CMA algorithm is proposed for wideband signals. The new algorithm is operating without the need for a reference signal such as the LMS algorithm. The results confirm that the proposed LMS-CMA scheme outperforms the conventional LMS and CMA algorithm. Simulation results LMS-CMA algorithm achieves better antenna array beamformation. The proposed array has $5 \mathrm{~dB}$ interference signals nulling higher compared to conventional LMS and CMA. The proposed LMS-CMA algorithm complexity is slightly higher than that of the LMS algorithm. 


\section{References}

[1] F.-B. Ueng, J.-D. Chen, and S.-H. Cheng, "Smart Antenna for Multiuser DS/CDMA Communication in Multipath Fading Channels," IEICE Transaction Communication, vol. E88, pp. 2944-2954, Jul 2005.

[2] J. A. Srar and K.-S. Chung, "Adaptive Array Beam Forming Using a Combined RLSLMS Algorithm," in APCC2008 Japan, 2008.

[3] T. Do-Hong and P. Russer, "Signal processing for wideband array applications," IEEE Microw., vol. 5, no. 1, pp. 57-67, Mar. 2004.

[4] B. Allen, M. Ghavami, "Adaptive Array Systems: Fundamentals and Applications," John Wiley \& Sons Ltd, England, 2005.

[5] D. Feng, Z. Bao, L. Jiao, "Total Least Mean Squares Algorithm", IEEE trans on signal proce, vol. 46, NO. 8, August 1998.

[6] M. Fujimoto, N. Kikuma, and N. Inagaki, "Performance of CMA adaptive array optimized by Marquardt method for suppressing multipath waves" (in Japanese), Trans. IEICE, vol. J74-B-II, no. 11, pp. 599-607, Nov. 1991. 